\title{
CPT-symmetry studies with antihydrogen
}

\section{Ralf Lehnert}

Published online: 24 January 2012

\begin{abstract}
Various approaches to physics beyond the Standard Model can lead to small violations of CPT invariance. Since CPT symmetry can be measured with ultrahigh precision, CPT tests offer an interesting phenomenological avenue to search for underlying physics. We discuss this reasoning in more detail, comment on the connection between CPT and Lorentz invariance, and review how CPT breaking would affect the (anti)hydrogen spectrum.
\end{abstract}

Keywords CPT-symmtery violation · antimatter · gravity

PACS 11.30.Er · 32.10.Fn · 04.30.-w

\section{Introduction}

Present-day established physics is well described by two different, mutually incompatible frameworks: quantum theory, which governs nature at the microscopic level, and classical general relativity, which dominates physical phenomena at large distance scales. Although the description of the physical world by these two theories is tremendously successful phenomenologically, the apparent necessity for two distinct frameworks is somewhat unsatisfactory from a theoretical perspective. For example, certain situations, such as the description of the birth of our universe, are characterized by conditions that call for the simultaneous application of both quantum theory and general relativity.

For this reason, significant efforts are presently directed towards a more fundamental description of nature that contains both quantum theory and classical general relativity as limiting cases. While there are a number of theoretical approaches in this context, phenomenological progress is hampered,

Ralf Lehnert

Indiana University Center for Spacetime Symmetries, Bloomington, IN 47405, USA

Tel.: +1-812-855-6190

Fax: +1-812-855-5533

E-mail: ralehner@indiana.edu 
primarily by the expected Planck suppression of quantum-gravity effects. A common avenue to tackle this issue is to look for model predictions that are not Planck suppressed. Examples are large extra dimensions or novel particles.

Another path towards quantum-gravity phenomenology is a bottom-up approach. It involves the identification of physical relations or physics principles testable with present-day or near-future technology at sensitivity levels that can be interpreted as having Planck reach. A further condition on such a relation or principle is that it should hold exactly in established physics, so that even the smallest observed deviations would definitely imply new physics. The third desirable feature is that at least some theoretical approaches to quantum gravity should allow for departures from the established physics in questions, so as to provide motivation for research efforts along these lines.

Spacetime symmetries satisfy the above three requirements: they can be studied experimentally with ultra-high precision, they hold exactly in known physics, and various theoretical ideas in the field of quantum gravity can accommodate their breakdown 11. Spacetime symmetries therefore represent an excellent area for research in this context.

Spacetime symmetries fall into two classes: continuous and discrete symmetries. The ten continuous ones are four translations, three rotations, and three Lorentz boosts. The discrete spacetime symmetry is CPT invariance. Note that these symmetries are closely intertwined. For example, in the context of axiomatic field theory with conventional quantum mechanics and local interactions, CPT violation goes hand in hand with Lorentz breaking [2].

In what follows, we will review the field-theory description of CPT violation at low energies and discuss its predictions for (anti)hydrogen spectroscopy as well as antihydrogen free-fall measurements.

\section{Field theory for CPT violation}

To test CPT invariance, it is desirable to employ a framework that allows for departures from this symmetry: such a framework places the identification and analysis of suitable CPT tests on a more solid footing. Various levels of $\mathrm{CPT}$ test models ranging from ad hoc assumptions of mass differences between particle and antiparticle to effective field theory (EFT) can in principle be considered. We proceed here at the level of EFT for the following reasons. EFT is a tremendously successful tool in various areas of physics including elementary-particle, nuclear, and condensed-matter physics. Note in particular that the latter area involves discrete backgrounds and non-relativistic dynamical aspects, features that may have to be modeled also in quantumgravity phenomenology. Moreover, EFT can implement CPT violation at the most fundamental level in established physics, which can guarantee internal theoretical consistency at all levels of presently known physics. In particular, an EFT model can include the usual Standard Model and general relativity, so that essentially all presently feasible CPT tests can be studied. 
Such an EFT framework for CPT violation - known as the Standard-Model Extension (SME) - is indeed available [3]. As per the aforementioned "AntiCPT Theorem" [2, CPT- and Lorentz-breaking are closely linked in the SME. Note, however, that even though all operators for departures from CPT invariance also violate Lorentz symmetry, the converse is not true.

The basic idea behind the inclusion of CPT and Lorentz breaking into the SME involves fixed, non-dynamical, tensorial structures that select preferred directions. More specifically, the SME effective Lagrangian $\mathcal{L}_{\mathrm{SME}}$ is

$$
\mathcal{L}_{\mathrm{SME}}=\mathcal{L}_{\mathrm{SM}}+\mathcal{L}_{\mathrm{EH}}+\delta \mathcal{L}_{\mathrm{SME}}
$$

where $\mathcal{L}_{\mathrm{SM}}$ and $\mathcal{L}_{\mathrm{EH}}$ denote the usual Standard-Model and Einstein-Hilbert terms, and $\delta \mathcal{L}_{\mathrm{SME}}$ contains small Lorentz- and CPT-violating contributions:

$$
\delta \mathcal{L}_{\mathrm{SME}}=i \bar{\psi} c^{\mu \nu} \gamma_{\mu} \partial_{\nu} \psi-\bar{\psi} a^{\mu} \gamma_{\mu} \psi+\ldots
$$

Here, $c^{\mu \nu}$ and $a^{\mu}$ are SME coefficients, where $a^{\mu}$ controls certain types of both Lorentz and CPT breakdown, while the Lorentz-violating $c^{\mu \nu}$ is CPT symmetric. Experimental studies seek to measure or constrain these (and other) tensorial coefficients.

The SME has been the framework for numerous experimental and phenomenological investigations of CPT and Lorentz invariance 4, including ones involving cosmic radiation [5], particle colliders [6], resonance cavities [7, neutrinos [8], and precision spectroscopy [9]. A number of theoretical analyses have studied various aspects of the internal consistency of the SME [10, but no problematic issues have been found thus far.

\section{Tests with exotic atoms}

Exotic atoms provide an excellent test ground for CPT and Lorentz symmetry for two reasons: the possibility for high-precision spectroscopy and the experimental access to types of matter difficult to study otherwise. The prospect for comparing matter to antimatter, and the associated potential for ultrasensitive CPT tests, is of particular interest in this context. In what follows, we will focus on two specific classes of tests, namely (anti)hydrogen spectroscopy and the interaction of antihydrogen with the gravitational field.

The effects of CPT and Lorentz violation on the spectrum of (anti)hydrogen within the minimal SME have been determined about a decade ago [11 and have previously been discussed at EXA 12 . It is therefore sufficient to give just a brief description of these results in the present work.

A natural candidate transition for CPT tests in (anti)hydrogen is the unmixed $1 \mathrm{~S}-2 \mathrm{~S}$ transition: the projected sensitivity is at the $10^{-18}$ level, which is promising in the context of Planck-scale tests. Unfortunately, the highly symmetric composition of these states eliminates first-order effects in the minimal SME. From a theoretical perspective, this transition is therefore less useful for CPT tests. Another option is a measurement of the spin-mixed $1 \mathrm{~S}-2 \mathrm{~S}$ transition. A study within the minimal SME does show that this transition would 
exhibit unsuppressed CPT violation. But there may be a practical disadvantage: the inhomogeneities in the trapping $B$ field would lead to a corresponding position dependence of the transition frequency, which represents a drawback for precision studies. A third idea would be the experimental determination of the hyperfine Zeeman splitting within the $1 \mathrm{~S}$ state itself. An analysis within the minimal SME indeed establishes unsuppressed CPT-breaking effects, and in principle, a magnetic-field independent transition point can be selected. Note in particular that similar transitions of this type, such as the usual Hydrogenmaser line, can be resolved with ultra-high precision.

The EFT description of the interplay between gravity and CPT and Lorentz violation is more involved than in the flat-spacetime limit. For example, the Bianchi identities become nontrivial on a curved manifold and impose conditions on the CPT- and Lorentz-violating background. One important theoretical result in this context is that an explicitly symmetry-breaking background is usually inconsistent 3 . A spontaneous violation of CPT and Lorentz invariance avoids such issues and is assumed in what follows.

For simplicity, we consider a sample scenario in which there is the only a single fermion of mass $m$, and the only non-zero SME coefficients are $a^{\mu}$ and $c^{\mu \nu}$. The corresponding terms in the Lagrangian are shown in Eq. (22). Our goal is now to extract the effective interaction of two point-like fermions in the non-relativistic limit for weak gravitational fields. This procedure requires various steps [13]. For instance, the aforementioned consistency condition of spontaneous symmetry breakdown requires the initial introduction of a further dynamical field. The dynamics of these additional degrees of freedom must then be interpreted correctly (i.e., they must be integrated out).

After various other considerations, and a further simplification to the isotropic case, only the two SME coefficients $a_{T}$ and $c_{T T}$ survive. The resulting two-parameter toy model is called the isotropic parachute model (IPM), and its classical non-relativistic point-particle Lagrangian reads [13]

$$
L_{\mathrm{IPM}}=\frac{1}{2} m_{\text {inertial }} v^{2}+\frac{m_{\text {grav }} M_{\text {grav }}}{r},
$$

where the SME coefficients are contained in the effective inertial and gravitational masses:

$$
m_{\text {inertial }}=m\left(1+\frac{5}{3} \bar{c}_{T T}\right), \quad m_{\text {grav }}=m+m \bar{c}_{T T} \pm 2 \alpha\left(\bar{a}_{\text {eff }}\right)_{T} .
$$

Here, $\bar{c}_{T T}$ and $\left(\bar{a}_{\text {eff }}\right)_{T}$ are effective coefficients for CPT and Lorentz breaking closely related to the original $a_{T}$ and $c_{T T}$. The \pm sign distinguishes between fermion and antifermion. The constant $\alpha$ depends on the details of the symmetry-violating field that needed to be introduced for consistency.

Lagrangian (3) is reminiscent of the conventional Lagrangian for a classical non-relativistic point particle in the gravitational field of a second point particle. All effects of CPT and Lorentz violation are absorbed into $m_{\text {inertial }}$ and $m_{\text {grav }}$. One can now consider various special regions in our toy model's two-dimensional $\left(m \bar{c}_{T T}, \alpha\left(\bar{a}_{\text {eff }}\right)_{T}\right)$ parameter space. For example, the special 
choice $\frac{1}{3} m \bar{c}_{T T}= \pm \alpha\left(\bar{a}_{\text {eff }}\right)_{T}$ means the following. For the + sign, the fermion behaves conventionally with $m_{\text {inertial }}=m_{\text {grav }}$, and free-fall modifications exist only for antifermions. For the other sign choice, the situation would be vice versa: novel effects are confined to matter, and antimatter behaves conventionally. The IPM toy model therefore illustrates that certain regions of the SME's parameter space are best tested with antimatter.

Acknowledgements The author wishes to thank the organizers for arranging this stimulating meeting and for the invitation to participate. This work is supported by the Indiana University Center for Spacetime Symmetries and the Portuguese Fundação para a Ciência e a Tecnologia under Grant No. CERN/FP/116373/2010.

\section{References}

1. See, e.g., V.A. Kostelecký and S. Samuel, Phys. Rev. D 39, 683 (1989); J. Alfaro, H.A. Morales-Técotl, and L.F. Urrutia, Phys. Rev. Lett. 84, 2318 (2000); S.M. Carroll et al., Phys. Rev. Lett. 87, 141601 (2001); J.D. Bjorken, Phys. Rev. D 67, 043508 (2003); V.A. Kostelecký et al., Phys. Rev. D 68, 123511 (2003); O. Bertolami et al., Phys. Rev. D 69, 083513 (2004).

2. O.W. Greenberg, Found. Phys. 36, 1535-1553 (2006).

3. D. Colladay and V.A. Kostelecký, Phys. Rev. D 58, 116002 (1998); V.A. Kostelecký and R. Lehnert, Phys. Rev. D 63, 065008 (2001); V.A. Kostelecký, Phys. Rev. D 69, 105009 (2004); V.A. Kostelecký and M. Mewes, Phys. Rev. D 80, 015020 (2009).

4. V.A. Kostelecký and N. Russell, Rev. Mod. Phys. 83, 11 (2011).

5. See, e.g., V.A. Kostelecký and M. Mewes, Phys. Rev. Lett. 87, 251304 (2001); Phys. Rev. D 66, 056005 (2002); R. Lehnert and R. Potting, Phys. Rev. Lett. 93, 110402 (2004); Phys. Rev. D 70, 125010 (2004); B.D. Altschul, Astropart. Phys. 28, 380 (2007); Phys. Rev. D 78, 085018 (2008).

6. See, e.g., M.A. Hohensee et al., Phys. Rev. Lett. 102, 170402 (2009); Phys. Rev. D 80, 036010 (2009); G. Amelino-Camelia et al., Eur. Phys. J. C 68, 619 (2010); J.P. Bocquet et al. [GRAAL Collaboration], Phys. Rev. Lett. 104, 241601 (2010); B.D. Altschul, Phys. Rev. D 80, 091901 (2009); Phys. Rev. D 84, 076006 (2011).

7. H. Müller et al., Phys. Rev. D 68, 116006 (2003); Ch. Eisele et al., Phys. Rev. Lett. 103, 090401 (2009); M.E. Tobar et al., Phys. Rev. D 80, 125024 (2009).

8. T. Katori et al., Phys. Rev. D 74, 105009 (2006); J.S. Díaz et al., Phys. Rev. D 80, 076007 (2009); V. Barger et al., Phys. Rev. D 84, 056014 (2011); J.S. Díaz and V.A. Kostelecký, Phys. Rev. D 85, 016013 (2012).

9. R. Bluhm et al., Phys. Rev. D 57, 3932 (1998); H. Dehmelt et al., Phys. Rev. Lett. 83 4694 (1999); R. Mittleman et al., Phys. Rev. Lett. 83, 2116 (1999); G. Gabrielse et al., Phys. Rev. Lett. 82, 3198 (1999); D. Bear et al., Phys. Rev. Lett. 85, 5038 (2000); P. Wolf et al., Phys. Rev. Lett. 96, 060801 (2006); I. Altarev et al., Phys. Rev. Lett. 103, 081602 (2009); Europhys. Lett. 92, 51001 (2010).

10. See, e.g., R. Jackiw and V.A. Kostelecký, Phys. Rev. Lett. 82, 3572 (1999); V.A. Kostelecký et al., Phys. Rev. D 65, 056006 (2002); B. Altschul and V.A. Kostelecký, Phys. Lett. B 628, 106 (2005); R. Lehnert, Phys. Rev. D 68, 085003 (2003); J. Math. Phys. 45, 3399 (2004); Phys. Rev. D 74, 125001 (2006); Rev. Mex. Fís. 56 (6), 469 (2010); A.J. Hariton and R. Lehnert, Phys. Lett. A 367, 11 (2007); D. Colladay and P. McDonald, Phys. Rev. D 77, 085006 (2008); Phys. Rev. D 79, 125019 (2009); M. Cambiaso et al., Phys. Rev. D 85, 085023 (2012).

11. R. Bluhm et al., Phys. Rev. Lett. 82, 2254-2257 (1999).

12. R. Lehnert, Hyperfine Interact. 193, 275 (2009).

13. V.A. Kostelecký and J. Tasson, Phys. Rev. Lett. 102, 010402 (2009); Phys. Rev. D 83, 016013 (2011). 